\title{
A Computational Evaluation of the Oxidative Cleavage of Triazine Derivatives for Electrosynthesis
}

\author{
Fergus J.M. Rogers and Michelle L. Coote* \\ ARC Centre of Excellence for Electromaterials Science, Research School of Chemistry, Australian National University, \\ ACT 2601, Australia
}

\begin{abstract}
Computational chemistry was used to study the N-C bond dissociation Gibbs free energies (298 K, acetonitrile) for a test set of 54 triazine derivatives, comprising combinations of 6 triazinyl radicals with 9 different alkyl leaving group. Results were obtained for homolytic cleavage of the neutral compound, and two possible mesolytic cleavage pathways for the oxidized form (cleavage to a triazinyl radical and a carbocation, or cleavage to a triazinyl cation and a carbon-centered radical). Oxidation potentials of the adducts and triazine radicals were also assessed. The aim of the study was to assess triazines as an alternative to alkoxyamines as electrochemical sources of carbon-centered radicals or cations. Oxidation potentials of the triazine adducts $\left(-0.1\right.$ to $+0.2 \mathrm{~V}$ vs $\left.\mathrm{Fc} / \mathrm{Fc}^{+}\right)$are lower than for corresponding alkoxyamines $(0.7-1.2 \mathrm{~V})$, making these compounds more functional group tolerant in electrosynthesis. In contrast to alkoxyamines, upon oxidation they generally cleave to a carbon-centered radical rather than a carbocation, and do so exergonically or at relatively low energies. Important exceptions are electron donating leaving groups for which mesolytic cleavage to a carbocation is preferred, though only in select cases is oxidative cleavage thermodynamically favored. The computational studies outlined herein support the possibility that an adduct based on the triazinyl radical is superior to an alkoxyamine for the electrochemical activation of carbon-centered radicals.
\end{abstract}

\section{INTRODUCTION}

The use of electrochemistry in place of chemical reagents in organic synthesis is currently gaining traction due to its atom economy and ability to control the production of highly reactive intermediates. ${ }^{1}$ Recently, we showed that electrochemical oxidation could be used to cleave alkoxyamines at room temperature so as to generate a clean source of carbocations for use in synthesis, polymerization and surface modification applications. ${ }^{2,3}$ Follow-up experimental and theoretical studies showed that the cleavage behavior could be altered by changing the leaving group and / or the solvent and electrolyte thus opening up a broad scope of $\mathrm{S}_{\mathrm{N}} 1, \mathrm{~S}_{\mathrm{N}} 2$ and radical reactions (Scheme 1). ${ }^{4,5}$

However, the oxidation potential for typical alkoxyamines is relatively high $(0.7-1.2 \mathrm{~V}$ vs $\mathrm{Fc} / \mathrm{Fc}+$ in acetonitrile ${ }^{4}$, which makes this method of carbocation or radical generation incompatible with some functional groups (e.g. aromatic amines, phenols, organosulfur compounds). Moreover, alkoxyamine synthesis and isolation can often be difficult or expensive. As a result, alternative substrates that can operate in a similar manner but without these disadvantages are desirable.

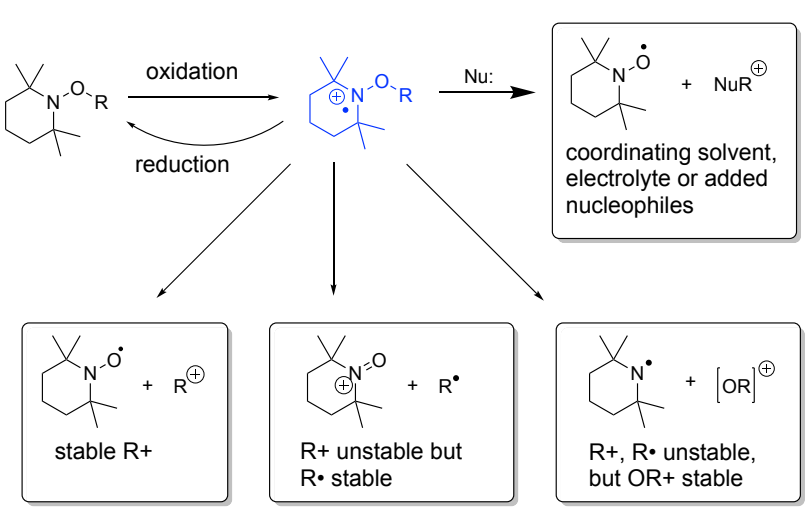

Scheme 1. Variation in oxidative cleavage of alkoxyamines according to leaving group and conditions.

Part of the driving force for the oxidative cleavage of alkoxyamines is the high inherent stability of the nitroxide radical and the associated low NO-R bond dissociation energy in the neutral form. As a result, we hypothesized that other $\mathrm{R}-\mathrm{X}$ compounds, where $\mathrm{X}$ is a stable radical might also be successful. Among these, benzo-1,2,4-triazinyl (Scheme 2) was first reported by Blatter in 1968 as an air and moisture stable radical. ${ }^{6}$ Benzo-1,2,4-triazinyl and its derivatives, known as triazinyls or Blatter radicals, can be easily prepared on a gram scale via methods suitable to derivatization, thus 
making it easy to tune their properties. ${ }^{7-10}$ This is important as substituent effects on the oxidation potentials of triazinyls are large and tunable. ${ }^{8}$ Like nitroxides, triazines have successfully been used as control agents in radical polymerization, further underlying their high inherent stability. ${ }^{11,12}$<smiles>CN1C(c2ccccc2)=NN(c2ccccc2)c2ccccc21</smiles>

Scheme 2. Blatter's radical, benzo-1,2,4-triazinyl

In the present work we use computational chemistry to evaluate whether alkyl-adducts of triazinyl radicals undergo oxidative cleavage to form carbon-centered radicals or carbocations under mild conditions, and to identify the most suitable derivatives for future experimental testing.

\section{COMPUTATIONAL PROCEDURES}

All calculations were carried out on full systems with no structural approximations or layering techniques applied. A preliminary benchmarking study was carried out to identify an accurate level of theory for these relatively large chemical systems (see Table S1, Supporting Information). Conformer searching was initially completed in acetonitrile with the economical B97D3BJ/6-31G(d) level of theory ${ }^{13,14}$ in conjunction with the SMD solvent model, ${ }^{15}$ as implemented in the Gaussian 16 software package. ${ }^{16}$ The lowest free energy geometries were relaxed in the gas-phase using the $\omega B 97 X-D / 6-31+G(d)$ level of theory, and all final geometries were confirmed as minima via vibrational analysis. ${ }^{17}$

Higher-level single point calculations were then performed using the new $\omega \mathrm{B} 97 \mathrm{M}(2)$ double-hybrid density functional developed of Mardirossian et al. ${ }^{18}$ This was completed in the latest revision of Q-Chem, version 5.1.1, ${ }^{19}$ using the recommended Ahlrichs def2-TZVPPD basis set with high-quality integration grids $(99,590)$. Perturbative corrections were determined via QChem's density fitting protocol. These improved gas phase free energies were then corrected to the solution phase using a thermocycle approach in conjunction with CPCM single point solvent corrections. ${ }^{20,21}$

Oxidation potentials $\left(\mathrm{E}_{\mathrm{ox}}\right)$ were calculated via the Nernst equation and referenced against the Fc/Fc+ couple in acetonitrile. To maximize error cancellation, an isodesmic method was used in which the experimental oxidation potential of $\mathrm{Ph}-\mathrm{tBu}$-Tri $(-0.27 \mathrm{~V})$ was used as a reference. ${ }^{22}$ Full details can be found in Table
S2 of the Supporting Information, which also details this protocol followed more completely.

\section{RESULTS AND DISCUSSION}

Test Set. To evaluate the feasibility of using triazines for oxidative cleavage, we chose a test set of 6 triazine adducts for each of 9 different leaving groups was considered (Scheme 3). For the triazines, substituents were chosen to include known compounds ${ }^{6-10,22}$ that could be easily synthesized and featured electron donating groups that were intended to stabilize the cation formed upon oxidation. A wide range of leaving $\mathrm{R}$ groups were selected, chosen to cover a range of radical and carbocation stabilities and to include type functionalities in synthesis and polymerization.

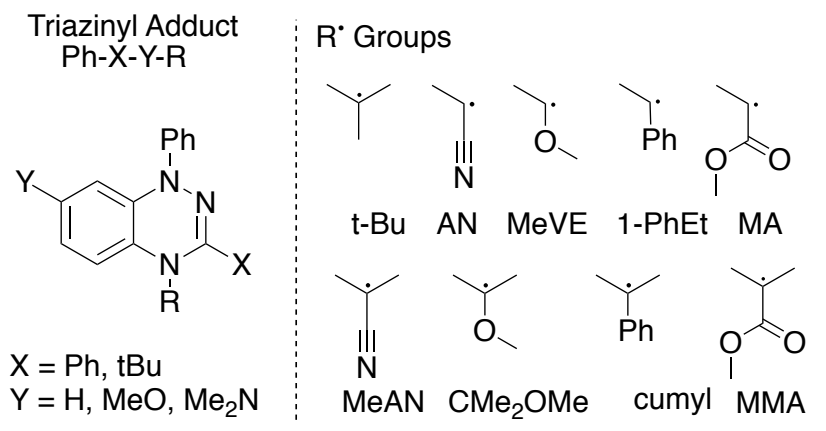

Scheme 3. Test in the present work

Oxidation. Oxidation potentials for the test set at $298 \mathrm{~K}$ in acetonitrile versus the $\mathrm{Fc} / \mathrm{Fc}+$ couple are shown in Table S2 of the Supporting Information. Figure 1 shows the potentials for each triazinyl radical, and average values for each triazine adduct (averaged over the same set of 9 R-groups in each case). This was possible as R-group trends within a given adduct are highly correlated with those within any other adduct (correlation coefficients $R=0.95-0.99$ ). Put another way, for the compounds studied, the oxidation potential of each triazine adduct is relatively unaffected by the R-group but depends heavily on the other triazine substituents.

As expected, within each series, the triazinyl radical oxidizes at a lower potential than its adduct. The oxidation potentials of the radicals are all very low, compared with, for example, nitroxide radicals and fall into the range of -0.7 to $-0.2 \mathrm{~V}$. The triazinyl adducts $(-0.1$ to $+0.2 \mathrm{~V}$ vs $\mathrm{Fc} / \mathrm{Fc}+$ ) also have low potentials compared with alkoxyamines $(0.7-1.2 \mathrm{~V}) .{ }^{4}$ This range is effectively outside of the oxidation window for most functional groups encountered in organic synthesis. In each case, the most easily oxidized species bear electron donating NMe2 groups to help stabilize the positive charge. For the radicals there is little difference between the $\mathrm{Ph}$ and $\mathrm{tBu}$ substituents as both are good 
electron donors, but for the adducts the phenyl groups are more effective at stabilizing the radical cation product.

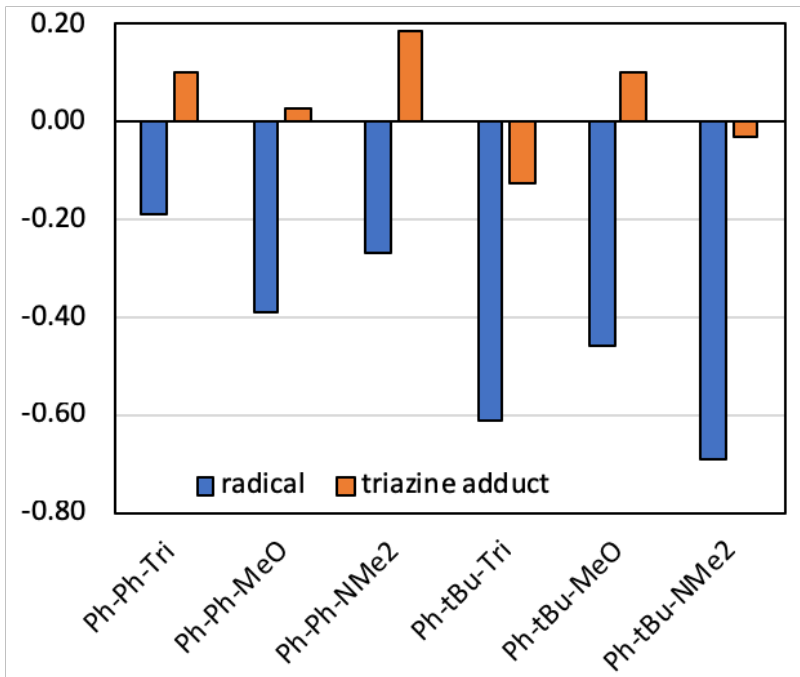

Figure 1. Oxidation potentials (298 K, acetonitrile, V vs $\mathrm{Fc} / \mathrm{Fc}+$ ) of the triazinyl radicals and their alkyl adducts. Values for the adducts were averaged over the same nine Rgroups in each case.

Oxidative Cleavage. To assess the potential for oxidative cleavage, $298 \mathrm{~K}$ bond dissociation Gibbs free energies were calculated for the neutral triazine-adducts and their oxidized counterparts. In the latter case both modes of mesolytic cleavage were considered: that producing a triazine radical and a carbocation, and that producing a triazine cation and a carbon-centered radical. The aim was to study spontaneous cleavage and so calculations were carried out in acetonitrile solution without considering the direct role of acetonitrile as a nucleophile in mediating cleavage via an $\mathrm{S}_{\mathrm{N}} 2$-type process. All 162 bond dissociation Gibbs free energies are provided in Table S3 of the Supporting Information and averages and correlation coefficients are provided in Table S4; key trends are summarized here.

Figure 2 shows the average Gibbs free energy for bond cleavage via each pathway for each of the triazines. The averages were taken over the same nine Rgroups in each series. Analysis of the individual data (Table S3 Supporting Information) showed that for a given triazine, the effects of R-group on the homolytic cleavage energy were highly correlated with the corresponding data for any other triazine studied (correlation coefficients $R=0.96-1.00$; Table S4 Supporting Information). Likewise, for mesolytic cleavage to a carbon-centered radical, the R-group trends for any one triazine were highly correlated with those for any other $(R=0.95-1.00)$, and the same was also true for mesolytic cleavage to carbocation $(R=1.00$; Table S4 Sup- porting Information). As a result, grouping the data together is instructive in comparing the different triazines.

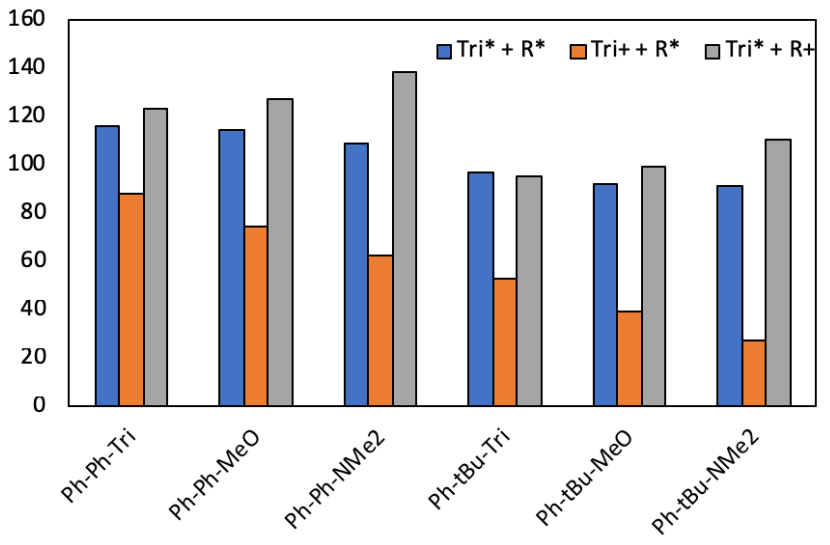

Figure 2. Effect of triazine on cleavage. Average (over the 9 R-groups of Scheme 3) Gibbs free energy (298 K, kJ mol-1, acetonitrile) for homolytic cleavage of neutral triazine adducts, and mesolytic cleavage of oxidized triazine adducts to form either carbon-centered radicals or carbocations.

However, as seen in Figure 2, the cleavage energies themselves are systematically shifted between the neutral and oxidized species by amounts that differ according to the triazine involved. For cleavage to a carboncentered radical, cleavage is promoted by oxidation but the extent to which it is favored in a given triazine reflects their differing ability to stabilize a radical versus a positive charge. The triazine most favored to undergo mesolytic cleavage to carbon-centered radicals is $\mathrm{Ph}$ tBu-NMe 2 , where the t-Bu and $\mathrm{NMe}_{2}$ are excellent electron donating groups for stabilizing the triazinyl carbocation.

Interestingly, the average cleavage to carbocations and triazine radicals are either competitive with or even disfavored when compared with the neutral species. This is in large part because these results are an average over several R-groups, many of which are unstable as carbocations. If instead we study the average R-group effects for different triazines (Figure 3) this becomes quite clear. While mesolytic cleavage to produce carbon-carbon-centered radicals is universally preferred over homolytic cleavage, albeit to differing extents according to the stability of the radical produced, for mesolytic cleavage to carbocation the results are much more variable. Thus, for species bearing electron withdrawing groups such as $\mathrm{C}(\mathrm{O}) \mathrm{OCH}_{3}$ or $\mathrm{CN}$ (i.e., that destabilize carbocations), the energies for mesolytic cleavage are increased compared with the neutral form. In all other cases energies are decreased, though it is only the species with strong electron donors (e.g. $\mathrm{OMe}$ ) that show thermodynamically preferred cleavage to carbocations rather than radicals. 


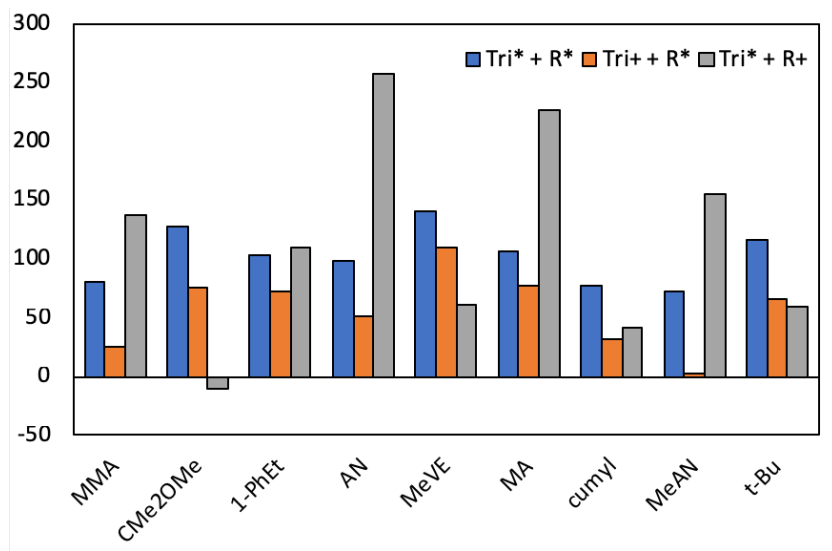

Figure 3. Effect of R-group on cleavage. Average (over the 6 triazines of Scheme 3) Gibbs free energy (298 K, kJ mol-1, acetonitrile) for homolytic cleavage of neutral triazine adducts, and mesolytic cleavage of oxidized triazine adducts to form either carbon-centered radicals or carbocations.
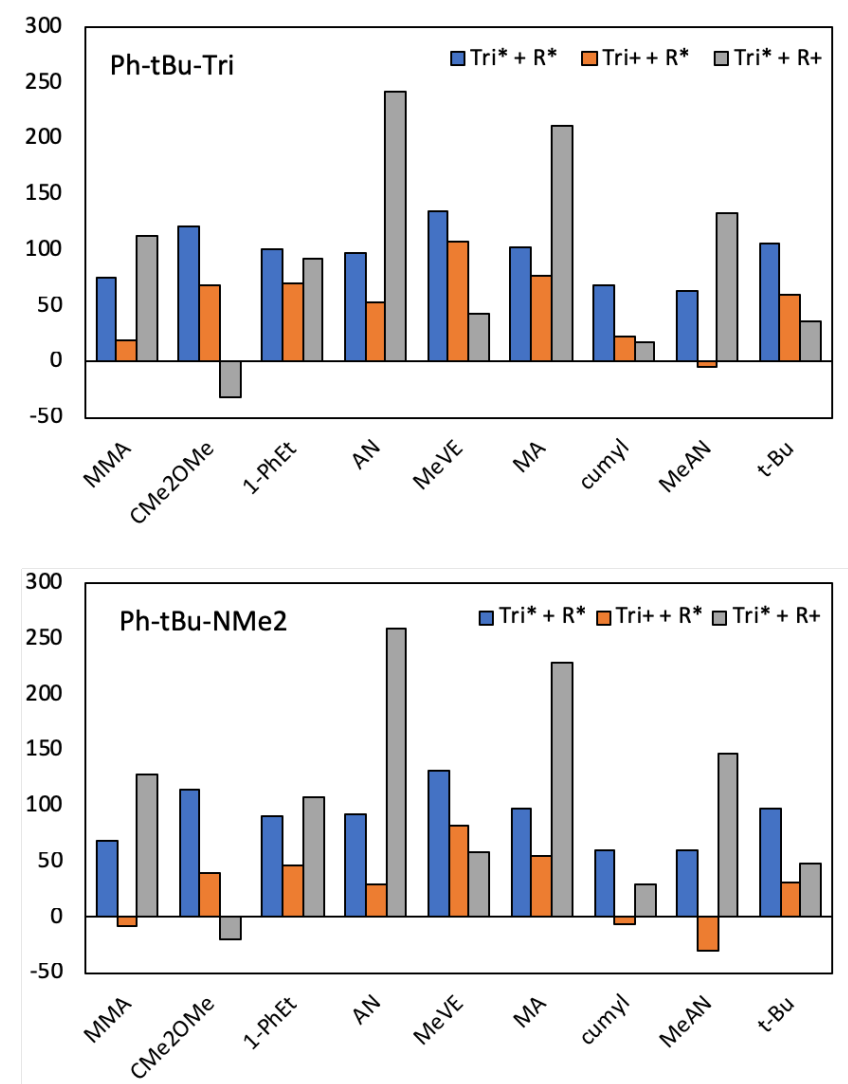

Figure 4. Gibbs free energy ( $298 \mathrm{~K}, \mathrm{~kJ} \mathrm{~mol}^{-1}$, acetonitrile) for homolytic cleavage of neutral triazine adducts, and mesolytic cleavage of oxidized triazine adducts to form either carboncentered radicals or carbocations. Top shows R-group effects for Ph-tB-Tri, while bottom shows R-group effects for Ph-tBuNMe2.

Design Guidelines. Putting this altogether, we can take Ph-tBu-Tri as the best triazine on average for car- bocation production, and $\mathrm{Ph}-\mathrm{tBu}-\mathrm{NMe}_{2}$ as the best triazine on average for radical production, and study the effects of R-group on the cleavage energy (Figure 4). For Ph-tBu-Tri, spontaneous mesolytic cleavage to a carbocation is thermodynamically favored for leaving group ${ }^{+} \mathrm{CMe}_{2} \mathrm{OMe}$ due to the strong electron donating methyl and methoxy groups. The energy, while positive, is also relatively low for other electron donating groups (MeVE, cumyl, tBu). For comparison, their mesolytic cleavage energies are all less than those calculated under equivalent conditions for the alkoxyamine TEMPO-iPr ${ }^{4}\left(66 \mathrm{~kJ} \mathrm{~mol}^{-1}\right)$, which has been shown to be reactive enough in its oxidized form to cleave via an $\mathrm{S}_{\mathrm{N}} 2$-type mechanism. ${ }^{5}$

If we instead focus on mesolytic cleavage to carboncentered radicals using $\mathrm{Ph}-\mathrm{tBu}-\mathrm{NMe}_{2}$, then MMA, cumyl and MeAN are all expected to cleave spontaneously to carbon-centered radicals. This is significant, as it implies that triazines could provide a room temperature, on demand source of radicals for polymerization and synthesis. For this triazine, mesolytic cleavage to a carbon-centered radical is also the preferred pathway for $\mathrm{t}-\mathrm{Bu}, \mathrm{MA}, \mathrm{AN}$ and 1-PhEt with Gibbs free energies less than $50 \mathrm{~kJ} \mathrm{~mol}^{-1}$, suggesting these would also be feasible as controlled radical sources with modest heating.

Carbocation Source

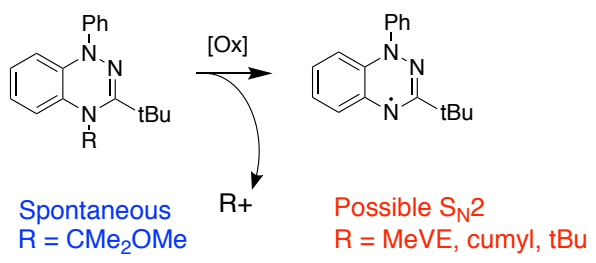

Radical Source

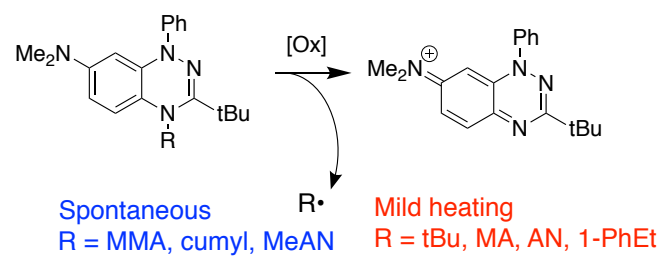

Scheme 4. Preferred structures for carbocation generation and carbon-centered radical production and their scope.

\section{CONCLUSIONS}

Triazinyl radicals, otherwise known as Blatter's radicals, are highly stabilized radicals that are easily synthesized and have low and highly tunable oxidation potentials. Here we show via computational chemistry that their adducts with alkyl radicals can undergo 
mesolytic cleavage upon oxidation in a manner analogous to alkoxyamines. Complementary to alkoxyamines, which tend to yield carbocations upon oxidative cleavage, triazine adducts show an increased preference for carbon-centered radical production, except when the leaving group is strongly electron donating. Triazine adducts also feature lower oxidation potentials than their alkoxyamine counterparts and hence should be more functional group tolerant. Based on our computational survey of 54 triazine derivatives the most ideal candidates for future experimental testing have also been identified (Scheme 4). Our work suggests that these species should expand the scope and utility of electrosynthesis in organic and polymer chemistry.

\section{ASSOCIATED CONTENT}

Supporting Information. Complete computational details. The supporting Information is available free of charge via the Internet at http://pubs.acs.org.

\section{REFERENCES}

1 Yan, M.; Kawamata, Y.; Baran, P. S. Synthetic Organic Electrochemical Methods Since 2000: On the Verge of a Renaissance, Chem. Rev. 2017, 117, 13230-13319.

2 Zhang, L.; Laborda, E.; Darwish, N.; Noble, B.B.; Tyrell, J.H.; Pluczyk, S.; Le Brun, A.P.; Wallace, G.G.; Gonzalez, J.; Coote, M.L., et al., Electrochemical and Electrostatic Cleavage of Alkoxyamines. J. Am. Chem. Soc. 2018, 140, 766-774.

3 Zhang, L.; Espíndola, R.; Noble, B.; Gonçales, V.; Wallace, G.; Darwish, N.; Coote, M.; Ciampi, S., Switchable Interfaces: Redox Monolayers On Si(100) by Electrochemical Trapping of Alcohol Nucleophiles. Surfaces 2018, 1, 3-11.

4 Hammill, C. L..; Noble, B. B.; Norcott, P. L.; Ciampi, S.; Coote, M, L., Effect of Chemical Structure on the Electrochemical Cleavage of Alkoxyamines. J. Phys. Chem. C 2019, 123, 5273-5281.

5 Noble, B. B.; Norcott, P. L.; Hammill, C. L..; Ciampi, S.; Coote, M, L., The Mechanism of Oxidative Alkoxyamine Cleavage: The Surprising Role of Solvent and Supporting Electrolyte, Submitted.

6 Blatter, H.M.; Lukaszewski, H., A New Stable Free Radical, Tetrahedron Lett., 1968, 22, 2701 - 2705.

7 Berezin, A.A.; Zissimou, G.; Constantinides, C.P.; Beldjoudi, Y.; Rawson, J.M.; Koutentis P.A., Route To Benzo- And PyridoFused 1,2,4-Triazinyl Radicals Via N' -(Het)Aryl-N' $-[2-\mathrm{Ni}-$ tro(Het)Aryl]Hydrazides J Org. Chem. 2014, 79, 314-327

8 Savva, A.C.; Zissimou, G.A.; Mirallai, S.I.; Berezin, A.A.; Demetriades, M.; Constantinides, C.P.; Nicolaides, C.; Trypiniotis, T.; Koutentis, P.A. Preparation of Blatter Radicals via Aza-Wittig Chemistry: The Reaction of $\mathrm{N}$ Arylimino-phosphoranes and 1(Het)aroyl-2-aryldiazenes, J. Org. Chem. 2017, 82, 7564-7575.

9 Bodzioch, A.; Zheng,M.; Kaszyński, P.; Utecht, G., Functional Group Transformations In Derivatives of 1,4-Dihydrobenzo[1,2,4]Triazinyl Radical J. Org. Chem. 2014, 79, 7294-7310

10 Grant, J.A.; Lu, Z.; Tucker, D.E.; Hockin, B.M.; Yufit, D.S; Fox, M.A; Kataky, R.; Chechik, V.; O’Donoghue, A.C., New BlatterType Radicals from a Bench-Stable carbene Nat. Commun. 2017 8, 15088

11 Demetriou, M. Berezin, A.A. Koutentis P.A. Krasia-Christoforou, T., Benzotriazinyl-Mediated Controlled Radical Polymerisation of Styrene Polym. Int., 2014, 63, 674-679.'

\section{AUTHOR INFORMATION}

\section{Corresponding Author}

* Email: michelle.coote@anu.edu.au

\section{Author Contributions}

The manuscript was written through contributions of all authors. / All authors have given approval to the final version of the manuscript.

\section{Funding Sources}

Australian Research Council (FL170100041)

\section{ACKNOWLEDGMENT}

MLC gratefully acknowledges a Georgina Sweet ARC Laureate Fellowship (FL170100041) and generous allocations of supercomputing time on the National Facility of the Australian National Computational Infrastructure.

12 Areephong, J.; Mattson, K.M.; Treat, N.J.; Poelma, S.O.; Kramer, J.W.; Sprafke, H.A.; Latimer, A.A.; Read De Alaniza, J.; Hawker, C.J., Triazine-Mediated Controlled Radical Polymerization: New Unimolecular Initiators, Polym. Chem., 2016, 7, 370 374.

13 Grimme, S. Semiempirical GGA-Type Density Functional Constructed with a Long-Range Dispersion Correction. J. Comput. Chem. 2006, 27, 1787-1799.

14 Grimme, S.; Ehrlich, S.; Goerigk, L. Effect of the Damping Function in Dispersion Corrected Density Functional Theory. J. Comput. Chem. 2011, 32, 1456-1465.

15 Marenich, A. V.; Cramer, C. J.; Truhlar, D. G. Universal Solvation Model Based on Solute Electron Density and on a Continuum Model of the Solvent Defined by the Bulk Dielectric Constant and Atomic Surface Tensions. J. Phys. Chem. B 2009, 113, 63786396

16 Frisch, M. J.; Trucks, G. W.; Schlegel, H. B.; Scuseria, G. E.; Robb, M. A.; Cheeseman, J. R.; Scalmani, G.; Barone, V.; Petersson, G. A.; Nakatsuji, H., et al., Gaussian 09 Rev. D.01, Wallingford, CT, 2016.

17 Chai, J.-D.; Head-Gordon, M. Long-Range Corrected Hybrid Density Functionals with Damped Atom - Atom Dispersion Corrections. Phys. Chem. Chem. Phys. 2008, 10, 6615-6620.

18 Mardirossian, N.; Head-Gordon, M. Survival of the Most Transferable at the Top of Jacob's Ladder: Defining and Testing the $\omega$ B97M(2) Double Hybrid Density Functional. J. Chem. Phys. 2018, 148, 241736

19 Shao, Y.; Gan, Z.; Epifanovsky, E.; Gilbert, A. T. B.; Wormit, M.; Kussmann, Lange, J. A. W.; Behn, A.; Deng, J.; Feng, X.; et al., Advances in Molecular Quantum Chemistry Contained in the QChem 4 Program Package. Mol. Phys. 2015, 113, 184-215.

20 Barone, V.; Cossi, M. Quantum Calculation of Molecular Energies and Energy Gradients in Solution by a Conductor Solvent Model. J. Phys. Chem. A 1998, 102, 1995-2001

21 Cossi, M.; Rega, N.; Scalmani, G.; Barone, V. Energies, Structures and Electronic Properties of Molecules in Solution with the C-PCM Solvation Model. J. Comput. Chem. 2003, 24, 669681 
1,2,4-Benzotriazin-4-Yl Radical. New J. Chem. 2015, 39, 4783-

22 Takahashi, Y.; Miura, Y.; Yoshioka, N. Synthesis and Properties 4789 .

of the 3-Tert-Butyl-7-Trifluoromethyl-1,4-Dihydro-1-Phenyl- 
TABLE OF CONTENTS GRAPHIC

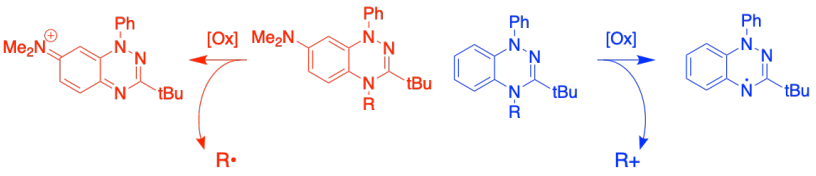

Stabilized Radical Source

Stabilized Carbocation Source 